\title{
Inverse spin-Hall effect in GeSn
}

\author{
A. Marchionni, ${ }^{1}$ C. Zucchetti, ${ }^{1, *}$ F. Ciccacci, ${ }^{1}$ M. Finazzi, ${ }^{1}$ H. S. \\ Funk $^{2}$ D. Schwarz, ${ }^{2}$ M. Oehme, ${ }^{2}$ J. Schulze, ${ }^{2}$ and F. Bottegoni ${ }^{1}$ \\ ${ }^{1}$ LNESS-Dipartimento di Fisica, Politecnico di Milano, \\ Piazza Leonardo da Vinci 32, 20133 Milano, Italy \\ ${ }^{2}$ Institute of Semiconductor Engineering (IHT), University of Stuttgart, Stuttgart, Germany
}

(Dated: January 22, 2021)

\begin{abstract}
We investigate the photoinduced inverse spin-Hall effect in a GeSn alloy with $5 \%$ Sn concentration. We generate a spin-polarized electron population at the $\Gamma$ point of the GeSn conduction band by means of optical orientation and we detect the inverse spin-Hall effect signal coming from the spinto-charge conversion in GeSn. We study the dependence of the inverse spin-Hall signal upon the kinetic energy of the spin-polarized carriers by varying the energy of the impinging photons in the $0.5-1.5 \mathrm{eV}$ range. We rationalize the experimental data within a diffusion model which explicitly accounts for momentum, energy and spin relaxation of the spin-polarized hot electrons. In this frame, at high photon energies, when the spin relaxation is mainly driven by phonon scattering, we extract a spin-Hall angle in GeSn which is more than ten times larger than the one of pure Ge. Moreover, the spin-charge interconversion for electrons lying at $\Delta$ valleys of GeSn results to be $\approx 4.3$ times larger than the one at $L$ valleys.
\end{abstract}

\section{INTRODUCTION}

Group IV semiconductors are the materials of choice of modern electronics. Therefore, the study of their spinrelated properties is extremely important in the perspective of developing spintronic devices compatible with this platform $[1,2]$. While spin-orbit phenomena are generally small in $\mathrm{Si}[3-5]$, Ge displays relevant spin-related effects occurring in the bulk [5-8] or at the surface [9] and at interfaces with other materials $[8,10,11]$. Moreover, typical spin-diffusion lengths in Ge are of the order of some micrometers [12-14] and the $4 \%$ lattice mismatch with $\mathrm{Si}$ allows for the implementation of Ge functionalities on the mainstream Si-based technology [15].

In this context, Sn can be a convenient route for the development of a platform with increased spin-orbit coupling (SOC) within group-IV materials. The absence of an energy gap in Sn does not allow field-effect operations. Anyway by alloying Ge with Sn atoms an energy gap is preserved while increasing the SOC of the lattice. Despite the small equilibrium solubility of $\mathrm{Sn}$ in Ge and the $14 \%$ lattice mismatch between the two materials [16, 17], the growth of high quality GeSn alloys on Si has been obtained [18]. This has paved the way to the demonstration of fundamental features from the optical and electronic point of view, such as the GeSn lasing action [19-21], or the possibility to increase the electron mobility as a function of the Sn concentration while still keeping efficient on-off transistor operations [22]. On the spintronic side, the large SOC in a group-IV semiconductor boost the interest in the spin-related properties of GeSn [23]. Since literature reports also suggest that the spin lifetime in GeSn lies in the nanoseconds range [24], this alloy could represent a novel platform where optoelectronic and spin-

\footnotetext{
* carlo.zucchetti@polimi.it
}

tronic architectures can be conveniently implemented.

In this work, we investigate the dependence of the spinHall angle, i.e., the efficiency of the spin-charge interconversion, as a function of the kinetic energy of the spinpolarized carriers of a $\mathrm{Ge}_{0.95} \mathrm{Sn}_{0.05}$ alloy. We generate a spin population by means of optical orientation [25-27] at the $\Gamma$ point of the GeSn conduction band, which undergoes spin-dependent scattering, generating an inverse spin-Hall effect (ISHE) electromotive field [28, 29]. The optically-injected spin current density $\mathbf{j}_{\mathrm{s}}$ is thus converted to a charge current density $\mathbf{j}_{\mathrm{c}}[28,29]$ :

$$
\mathbf{j}_{\mathrm{c}}=\gamma \mathbf{j}_{\mathrm{s}} \times \mathbf{u}_{\mathrm{P}}
$$

being $\gamma$ the spin-Hall angle and $\mathbf{u}_{\mathrm{P}}$ the unit vector representing the direction of the spin-polarization. Experimentally, we detect the voltage drop $\Delta V_{\text {ISHE }}$ due to $\mathbf{j}_{\mathrm{c}}$ [11] as a function of the incident photon energy across two electrodes at the edges of a GeSn bar, and characterize the spin transport properties of hot electrons [7]. From the measured signal and exploiting a model explicitly accounting for momentum, spin and energy relaxation of hot electrons, we infer the spin-charge interconversion efficiency $\gamma$ as a function of the kinetic energy of the carriers. For incident photon energies much larger than the direct GeSn gap, the spin-dependent scattering is dominated by phonons and we estimate $\gamma \approx 0.3$, a value ten times larger than the one obtained in bulk $\mathrm{Ge}$ [7]. Moreover, in GeSn we obtain a different efficiency of spin-charge interconversion for electrons diffusing at the $L$ and $\Delta$ valleys, the latter being a factor 4.3 larger than the former.

\section{METHODS}

The investigated sample is a single crystalline $n$ doped $\mathrm{Ge}_{0.95} \mathrm{Sn}_{0.05}$ stripe, grown on a $525 \mu \mathrm{m}$-thick 


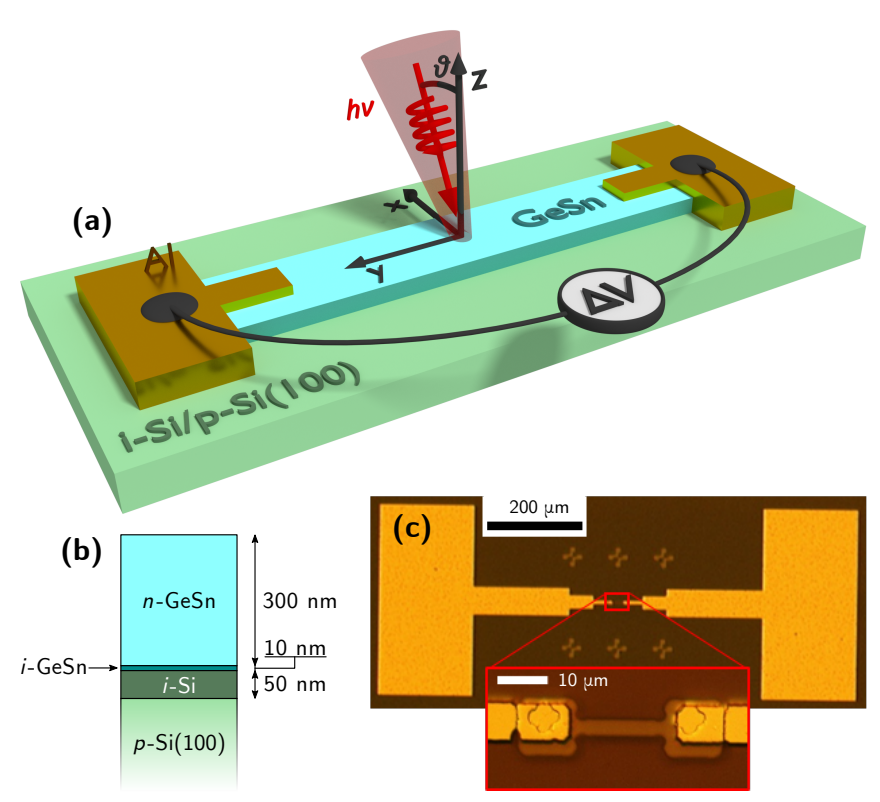

Figure 1. (Color online) Sample and experimental geometry. (a) A single crystalline $\mathrm{Ge}_{0.95} \mathrm{Sn}_{0.05}$ stripe of dimensions $d_{x} \times d_{y} \times d_{z}=5 \times 20 \times 0.3 \mu \mathrm{m}^{3}$ lies on top of a Si substrate. From two Al pads deposited onto the stripe we measure the voltage drop $\Delta V_{\text {ISHE }}$ due to the photoinduced ISHE. A focused circularly-polarized light beam shine the sample with polar angle $\vartheta$. (b) The molecular-beam epitaxy grown stack with doped GeSn layer on Si. (c) Optical image of the investigated sample and zoom (red inset) on GeSn bar.

Si substrate, as sketched in Fig. $1(\mathrm{a}, \mathrm{b})$. The GeSn is doped with antimony with concentration $N_{\mathrm{d}}=7.4 \times 10^{17} \mathrm{~cm}^{-3}$, while the substrate has a boron doping $N_{\mathrm{a}} \sim 10^{16} \mathrm{~cm}^{-3}$. The geometrical dimensions of the stripe are $5 \mu \mathrm{m}, 20 \mu \mathrm{m}$ and $300 \mathrm{~nm}$ along the $x, y$ and $z$ axis, respectively, within the reference frame of Fig. 1(a). An optical image of the entire investigated structure is shown in Fig. 1 (c) (see Appendix A for complete growth and fabrication details).

The geometry of the measurements is illustrated in Fig. 1 (a). We exploit a supercontinuum laser [30] as a light source, monochromatized with a typical bandwith of $\approx 10 \mathrm{meV}$ and tuned in the $0.5-1.5 \mathrm{eV}$ energy range. The circular-polarization of the light beam is modulated at $50 \mathrm{kHz}$ by a photoelastic modulator (PEM). The light beam is focused on the sample with a polar angle $\vartheta \approx 30^{\circ}$, by partially filling out-of-axis an objective with $0.7 \mathrm{nu}-$ merical aperture. This results in the generation of a spin-polarization $\mathbf{P}=P \mathbf{u}_{\mathrm{P}}$ with a non-vanishing component along the $x$-axis, since $\mathbf{u}_{\mathrm{P}}$ is antiparallel to the light wavevector inside GeSn. The electrical ISHE signal is acquired as a voltage drop $\Delta V_{\text {ISHE }}$ (along $y$ ) between two $\mathrm{Al}$ electrodes and is demodulated with a lock-in amplifier at the PEM frequency. An off-normal illumination of the sample is fundamental since in our geometry the optically-injected spin current $\mathbf{j}_{\mathrm{s}}$ diffuses along the $z$ axis [7], whereas the detected charge current $\mathbf{j}_{\mathrm{c}}$ flows along $y$ :


Figure 2. (color online) Experimental data. (a,b) Dependence of $\Delta V_{\text {ISHE }}$ on the degree of circular polarization (DCP) and on the polar angle $\vartheta$, respectively, of the impinging light beam. Dotted line show linear fits of the data. (c) Photon energy dependence of $\Delta V_{\text {ISHE }}$ normalized to the flux of photons that are transmitted to GeSn. The inset show a zoom of the experimental data in the $0.6 \div 1.15 \mathrm{eV}$ region.

therefore, according to Eq. (1) the projection of $\mathbf{P}$ along $x$ is the only detectable component of the spin polarization. To further increase the signal-to-noise ratio, the light intensity is chopped at $1.12 \mathrm{~Hz}$ and the ISHE signal is then extracted by a second lock-in amplifier in cascade with the first one. All the measurements are performed at room temperature.

\section{EXPERIMENTAL RESULTS}

Fig. 2 (a) shows the dependence of the ISHE signal upon the degree of circular polarization (DCP) of the impinging light beam. This is achieved by varying the phase delay of the PEM, as detailed in the Supplemental Material of Ref. 31. The observed linear dependence is in agreement with calculations performed by means of multilayer optical analysis [32] related to the photoinduced ISHE. In Fig. 2 (b) we show the dependence of $\Delta V_{\text {ISHE }}$ as a function of the polar angle $\vartheta$, defined in Fig. 1 (a). The $x$-component of the spin polarization $\mathbf{P}$ is opposite for opposite $\vartheta$ values, thus yielding an opposite ISHE signal. Moreover, the ISHE signal is linear with $\vartheta$, as expected 

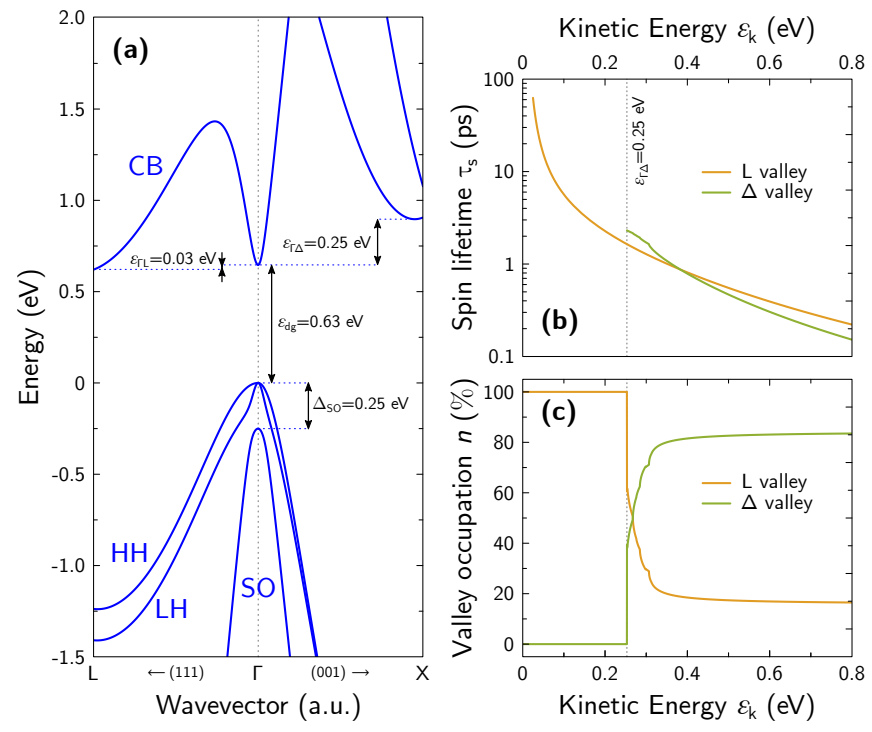

Figure 3. (color online) Theoretical calculations. (a) Band structure of GeSn with 5\% Sn concentration, calculated within a full-band $\mathbf{k} \cdot \mathbf{p}$ model [16, 33]. (b,c) Kinetic energy dependence of the spin lifetime (b) and the probability of valley occupation (c), obtained from the model of Ref. 7.

for small angles inside the GeSn sample (in our configuration $\left.\vartheta_{\mathrm{GeSn}}<7^{\circ}\right)$ [32]. The datasets of Fig. $2(\mathrm{a}, \mathrm{b})$ confirm the spin-related nature of the electrical signal acquired with our experimental setup.

Finally, the dependence of $\Delta V_{\text {ISHE }}$ as a function of the incident photon energy is reported in Fig. 2 (c). The experimental data are normalized to the flux of photons $\Phi_{\mathrm{ph}}$ transmitted into GeSn. For the sake of clarity, a zoom of the ISHE signal in the $0.6-1.15 \mathrm{eV}$ range is shown in the inset: in this case it is possible to observe that the signal is positive for photon energies larger than $0.7 \mathrm{eV}$ and it changes sign at $h \nu \approx 1.07 \mathrm{eV}$. At larger photon energies the signal rapidly increases, scaling almost linearly with $h \nu$. In the following, we will refer to $\varepsilon_{\mathrm{SR}}=1.07 \mathrm{eV}$ as the photon energy corresponding to the sign reversal in the ISHE spectrum.

\section{PHYSICAL PICTURE}

In the investigated photon energy range, optical transitions around the $\Gamma$ point of the GeSn Brillouin zone can promote electrons from the heavy-hole $(\mathrm{HH})$, lighthole ( $\mathrm{LH})$ and $\mathrm{SO}$ branches into the conduction band, as shown in Fig. 3(a), which displays the band structure of GeSn with 5\% Sn concentration, calculated with a full $\mathbf{k} \cdot \mathbf{p}$ model $[16,33]$. If the semiconductor is illuminated with a circularly polarized light beam, the spinpolarization of electrons excited from the $\mathrm{HH}$ branch is opposite compared to the one of electrons promoted from LH and SO bands. Moreover, the intensity of the transitions from $\mathrm{HH}, \mathrm{LH}$, and $\mathrm{SO}$ have a relative weight of 3 ,
1 , and 2 , respectively. This, combined with a larger joint density of states for the $\mathrm{HH} \rightarrow \mathrm{CB}$ transitions, produces a net spin-polarization $P$ at the generation time mostly given by electrons promoted from $\mathrm{HH}$ within the whole investigated photon energy range. This has already been demonstrated in Ge [34], and is further discussed in Appendix B for GeSn. Since $P=50 \%$ if the incident photon energy is tuned to the direct GeSn gap and monotonically decreases down to zero when the photon energy is increased [34], the detected sign reversal in the ISHE spectrum cannot be ascribed to the energy dependence of the injected spin polarization.

The key ingredient to explain the experimental results of Fig. 2 (c) is the dependence of the electron spin lifetime $\tau_{\mathrm{s}}$ as a function of the kinetic energy $\varepsilon_{\mathrm{k}}$, which is reported in Fig. 3 (b). Following the procedure detailed in Ref. $7, \tau_{\mathrm{s}}$ has been calculated by accounting for the momentum scattering due to impurities and phonons (intraand inter-valleys for all the valleys), related to electrons in the $L$ and $\Delta$ valleys. Then, the energy dependence of the spin relaxation has been evaluated by means of the Yafet-Elliott cross section [35].

Similarly to the case of Ge, for $\varepsilon_{\mathrm{k}} \lesssim 0.2 \mathrm{eV}$ momentum (and spin) relaxation is mainly driven by impurities, while phonons dominate scattering at higher kinetic energies [7]. Due to energy conservation, electrons from the $\mathrm{HH}$ band are always promoted to higher energies in the $\mathrm{CB}$ compared to electrons coming from the SO branch. According to the results of Fig. 3 (b), this means that the former experience a spin lifetime much shorter than the latter. Hence, the spin polarized electron population coming from the $\mathrm{HH}$ band determines the spin polarization $P$ at the generation time. However, such a population rapidly depolarizes and, under steady-state conditions, the spin character of the entire electron population is given mostly by spins excited from SO states. This explains the sign reversal at the onset of the optical transitions from SO, and nicely fits the ISHE experimental data acquired in Ge [7].

In the case of $\mathrm{GeSn}$ the onset of the optical transitions from the $\mathrm{SO}$ band is $h \nu=0.88 \mathrm{eV}$, while the sign reversal is observed at $\varepsilon_{\mathrm{SR}}=1.07 \mathrm{eV}$. Such an energy shift can be explained in a frame which accounts not only the dependence of $\tau_{\mathrm{s}}$ upon $\varepsilon_{\mathrm{k}}$, but also the kinetic energy dependence of the valley occupation at the steady state. In Fig. 3 (c) the occupation probability $n_{L}\left(n_{\Delta}\right)$ of the $L(\Delta)$ valleys as a function of $\varepsilon_{\mathrm{k}}$ is shown, assuming $n_{\Gamma}=0$, and exploiting the model of Ref. 7. The occupation of the $\Gamma$ valley can be set to zero due to the negligible density of states of $\Gamma$ compared to $L$ and $\Delta$. From Fig. 3 (c) if electrons have enough kinetic energy to reach $\Delta$, they are favored to populate this valley. This is a consequence of the larger density of states of $\Delta$ compared to $L$. Therefore, a larger electron kinetic energy is related to the decrease of the spin lifetime [see Fig. 3 (b)] and the increase of the population at $\Delta$ compared to $L$ [see Fig. 3 (c)]. Notably, electrons coming from HH states start populating the $\Delta$ valley starting from a photon en- 

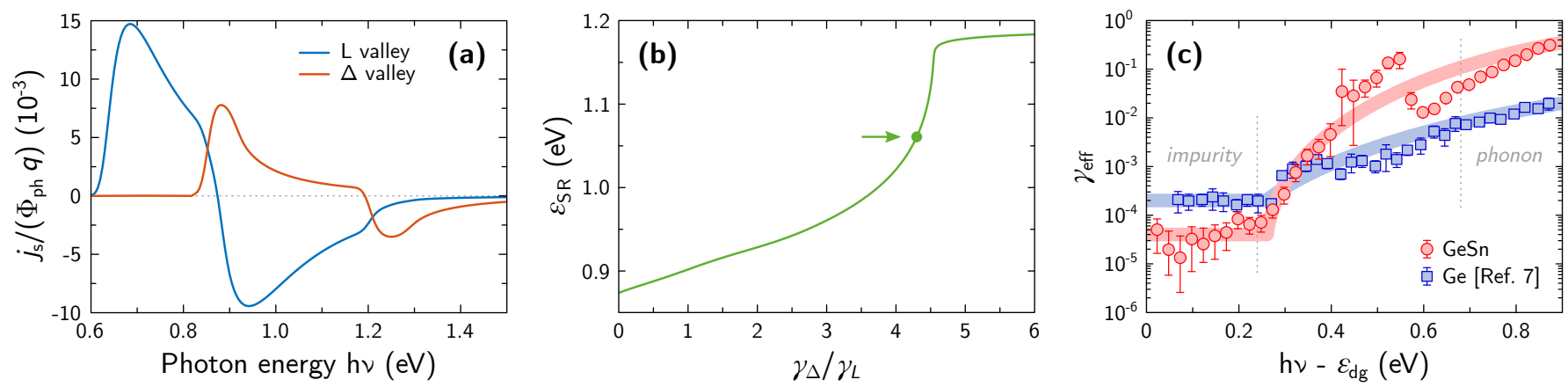

Figure 4. (color online) Physical picture. (a) Calculation of the spin current density flowing in $L$ and $\Delta$ valleys as a function of the photon energy. Results are convoluted with a Gaussian function describing both the thermal broadening of the states $(\approx 26 \mathrm{meV})$ and the bandwidth of the light source $(\approx 10 \mathrm{meV})$. (b) Photon energy of the sign reversal $\varepsilon_{\mathrm{SR}}$ as a function of the relative weight of the spin-Hall angles of electrons in $\Delta\left(\gamma_{\Delta}\right)$ and $L\left(\gamma_{L}\right)$. The arrow correspond to the experimental $\varepsilon_{\mathrm{SR}}$ value. (c) Estimated effective spin-Hall angle (see text) for GeSn (red circles) and Ge (blue squares, data from Ref. 7). The solid lines are guides for the eyes. Datasets are aligned to the direct gap energy of the materials.

$\operatorname{ergy} h \nu \approx \varepsilon_{\mathrm{dg}}+\varepsilon_{\Gamma \Delta}=0.88 \mathrm{eV}$ [see Fig. $\left.3(\mathrm{a})\right]$ which also correspond to the onset of the transitions from the $\mathrm{SO}$ branch. Hence, for photon energies larger than $0.88 \mathrm{eV}$, spin-polarized electrons excited from $\mathrm{HH}$ diffuses in $\Delta$, while the electrons with opposite spin polarization promoted from the SO states are thermalized and diffuse within the $L$ valley.

As previously observed in GaAs [36, 37], the spin transport in different valleys can be associated to different spin-charge interconversion efficiencies. The reason of the shift of $\varepsilon_{\mathrm{SR}}$ to higher energies with respect to the onset of the optical transitions from SO lies in a larger spinHall angle of electrons occupying $\Delta$ states with respect to those lying in the $L$ valley. Therefore, electrons diffusing along $\Delta$ provides for a larger contribution to the ISHE signal. Thus, for $h \nu>0.88 \mathrm{eV}$, although electrons coming from $\mathrm{HH}$ have a lower electron spin lifetime due to the larger kinetic energy, the diffusion along $\Delta$ ensures a larger spin-to-charge conversion with respect to the electron population excited from the SO and lying at $L$. In conclusion, the sign reversal of the ISHE signal is dictated by the trade-off between the energy dependence of the electron spin lifetime and the valley-dependent spin-Hall angle. This produces the shift to larger photon energies of $\varepsilon_{\mathrm{SR}}$.

\section{DISCUSSION}

We separately calculate the spatial average along the stripe thickness of the steady-state spin current densities at the $\Delta$ and $L$ valleys, i.e., $j_{\mathrm{s}}^{L}$ and $j_{\mathrm{s}}^{\Delta}$, respectively. The results are reported in Fig. 4(a), see Appendix C for details. Due to ISHE the spin current densities $j_{\mathrm{s}}^{L}$ and $j_{\mathrm{s}}^{\Delta}$ generate a flow of charges in $L\left(j_{\mathrm{c}}^{L}\right)$ or in $\Delta$ $\left(j_{\mathrm{c}}^{\Delta}\right)$, each characterized by a spin-charge interconversion efficiency $\gamma_{L}$ or $\gamma_{\Delta}$, respectively. Both charge currents then contribute to the measured $\Delta V_{\text {ISHE}}$. Since for either
$L$ and $\Delta$ valleys $\left|\mathbf{j}_{\mathrm{s}} \times \mathbf{u}_{P}\right|=j_{\mathrm{s}} \sin \vartheta_{\mathrm{GeSn}}$, from Eq. (1) the total ISHE signal can be expressed as:

$$
\Delta V_{\mathrm{ISHE}}=\left(\gamma_{L} j_{\mathrm{s}}^{L}+\gamma_{\Delta} j_{\mathrm{s}}^{\Delta}\right) R d \Phi_{\mathrm{ph}} \sin \vartheta_{\mathrm{GeSn}},
$$

being $R \approx 23 \mathrm{k} \Omega$ the stripe resistance [7,36-38]. The sign reversal of $\Delta V_{\text {ISHE }}$ is related to a sign reversal of the term in the brakets. However, $j_{\mathrm{s}}^{L}$ and $j_{\mathrm{s}}^{\Delta}$ become negative for different photon energies [see Fig. 4 (a)]: as a consequence, we can exploit the ratio $\gamma_{\Delta} / \gamma_{L}$ as a free parameter to tune $\varepsilon_{\mathrm{SR}}$ to the experimentally detected value $\varepsilon_{\mathrm{SR}}=1.07 \mathrm{eV}$ [see Fig. 2 (c)]. In this case, we obtain $\gamma_{\Delta} / \gamma_{L} \approx 4.3$, meaning that the spin-orbit scattering of spin-polarized electrons lying at the $\Delta$ valleys is almost 4.3 times more effective compared to the one of electrons lying at the $L$ valleys. This value results to be robust against reasonably small variations of the input parameter for the calculation. For the sake of clarity, in Fig. 4 (b) we show the dependence of $\varepsilon_{\mathrm{SR}}$ (calculated as the photon energy by which $\left.\gamma_{L} j_{\mathrm{s}}^{L}+\gamma_{\Delta} j_{\mathrm{s}}^{\Delta}=0\right)$ as a function of the ratio between the spin-charge interconversion efficiencies $\gamma_{\Delta} / \gamma_{L}$

A macroscopic spin-to-charge conversion parameter $\gamma_{\text {eff }}$ can be extracted by weighting each valley-dependent spin-charge interconversion efficiency with the valley occupation [37]:

$$
\gamma_{\mathrm{eff}}=\gamma_{L} n_{L}+\gamma_{\Delta} n_{\Delta} \approx \gamma_{L}\left(n_{L}+4.3 n_{\Delta}\right) .
$$

The result is reported in Fig. 4 (c), where we compare $\gamma_{\text {eff }}^{\text {GeSn }}$ with the spin-Hall angle of Ge $\gamma_{\text {eff }}^{\text {Ge }}$, reproduced from Ref. 7. The data are aligned to the photon energy corresponding to the direct energy gap $\varepsilon_{\mathrm{dg}}$. Note that the drop of $\gamma_{\mathrm{eff}}^{\mathrm{GeSn}}$ at $h \nu-\varepsilon_{\mathrm{dg}} \approx 0.55$ corresponds to the peak in $j_{\mathrm{s}}^{\Delta}$ at $1.25 \mathrm{eV}$ [see Fig. 4(a)], which could be overestimated due to the parabolic approximation of the bandstructure within the employed model.

For photon energies close to $\varepsilon_{\mathrm{dg}}$, where the spindependent scattering is mostly driven by impurities, the 
spin-charge interconversion is almost constant within the experimental error and $\gamma_{\text {eff }}^{\mathrm{GeSn}} \approx 4 \times 10^{-5}$, while $\gamma_{\text {eff }}^{\mathrm{Ge}} \approx 2 \times 10^{-4}$. In this energy range the efficiency of spin-dependent scattering is determined by the SOC of the dopant (according to the atomic number $Z$ ) and by the doping concentration. Despite GeSn is Sbdoped $\left(Z_{\mathrm{Sb}}=51\right)$ while $\mathrm{Ge}$ is $\mathrm{P}$-doped $\left(Z_{\mathrm{P}}=15\right)$, $\gamma_{\text {eff }}^{\mathrm{Ge}}>\gamma_{\text {eff }}^{\mathrm{GeSn}}$ due to the large difference in the number of dopants $\left(N_{\mathrm{Sb}}=7.4 \times 10^{17} \mathrm{~cm}^{-3}, N_{\mathrm{P}}=2 \times 10^{19} \mathrm{~cm}^{-3}\right)$. This strongly reduces the cross section of the impurities in GeSn compared to the Ge sample. Moreover, in thin GeSn films the large lattice mismatch between $\mathrm{Si}$ and GeSn leads to defect-rich material [18], which contribute to further decrease the spin-Hall angle.

As observed in $\mathrm{Ge}$, the spin-charge interconversion is drastically enhanced as the phonon-contribution to spindependent scattering increases [7]. Here, the spin-Hall angle of GeSn becomes larger than the one of Ge [see Fig. $4(\mathrm{c})$ ]. Indeed, the phonon-mediated electron scattering mostly depends on the SOC of the lattice rather than the one of the scattering centers such as impurities, and, compared to pure Ge, the SOC of GeSn is increased by the presence of Sn. The largest spin-to-charge conversion efficiency for GeSn is $\gamma_{\text {eff }}^{\mathrm{GeSn}} \approx 0.3$, at least ten times larger than the one of Ge.

\section{CONCLUSION}

We have exploited optical orientation to generate a spin-polarized electron population in the conduction band of GeSn. We have measured the inverse spin-Hall effect signal due to the spin-dependent scattering taking place in GeSn itself and we have exploited a spin diffusion model based on the kinetic energy dependence of the spin-relaxation time to reproduce the observed ISHE spectrum. The spin-Hall angle of GeSn is more than a factor ten larger than the one of Ge, when spin-dependent scattering is mediated by phonons, due to the larger spinorbit coupling of the GeSn lattice compared to Ge.

\section{Appendix A: Growth and farbrication of GeSn}

All substrates are grown using solid source molecular beam epitaxy (SS-MBE). Si is evaporated using an electron beam evaporator and Ge, Sn and Sb are evaporated using resistively heated Knudsen-type cells. The Ge and Si rates are measured and controlled using a quadrupole mass spectrometer (QMS) and fixed to a rate $1 \AA \cdot \mathrm{s}^{-1}$. The $\mathrm{Sn}$ and $\mathrm{Sb}$ rates are controlled through the cell temperature using a thermocouple. The Sn and Sb cell temperatures are calibrated to the required rates. The Sn rate is adjusted to achieve the required $\mathrm{Ge}_{0.95} \mathrm{Sn}_{0.05}$ composition, resulting in a total $\mathrm{Ge}_{0.95} \mathrm{Sn}_{0.05}$ growth rate larger than $1 \AA \cdot \mathrm{s}^{-1}$. In the same manner, the $\mathrm{Sb}$ flux is adjusted for each sample to achieve the required $\mathrm{Sb}$ doping concentration. The growth is performed on in- dustry standard $p$ - $\mathrm{Si}(100)$ substrates with a specific resistance of $10-20 \Omega \cdot \mathrm{cm}$. After sample loading, the wafers are annealed at a substrate temperature of $900^{\circ} \mathrm{C}$ for 5 min to remove the native $\mathrm{SiO}_{2}$ [39]. Afterwards, the growth starts with an intrinsic Si layer with a thickness of $50 \mathrm{~nm}$ at $600^{\circ} \mathrm{C}$ to overgrow any surface imperfection. The sample temperature is then reduced to $160^{\circ} \mathrm{C}$ for the subsequent $\mathrm{Ge}_{0.95} \mathrm{Sn}_{0.05}$ growth. An initial intrinsic $\mathrm{Ge}_{0.95} \mathrm{Sn}_{0.05}$ wetting layer with thickness $10 \mathrm{~nm}$ is grown. Since no Sb segregation is expected for $\mathrm{Ge}(100)$ at $160^{\circ} \mathrm{C}$ [40], the $\mathrm{Sb}$ shutter is opened when starting the growth of the final $n$-Ge $\mathrm{Ge}_{0.95} \mathrm{Sn}_{0.05}$ layer with $d_{z}=300 \mathrm{~nm}$ to enable doping via co-evaporation. The resulting layer stack is shown in Fig. 1(b). The samples are cleaned in acetone and isopropanol ultrasonic bath for $8 \mathrm{~min}$ and 3 min, respectively (standard Ge clean). The structures are defined using optical lithography. Immediately before photoresist spin-on, all samples are cleaned in $\mathrm{HCl}$ with concentration $3 \mathrm{~mol} \cdot \mathrm{l}^{-1}$ to remove the native oxide and then coated with hexamethyldisilazane (HMDS) adhesion promoter to avoid photoresist liftoff.

All structures are etched using HBr by means of inductively coupled plasma reactive ion etching (ICP-RIE). The whole MBE grown stack is etched to achieve freestanding structures that are electrically insulated from their surroundings. After etching, samples are resist stripped using TMAH, followed by a standard Ge clean and $\mathrm{O}_{2}$ plasma clean. The samples are coated with low temperature $\mathrm{SiO}_{2}$ with $300 \mathrm{~nm}$ grown by plasma enhanced chemical vapor deposition (PECVD) at $250^{\circ} \mathrm{C}$ using tetraethoxysilane (TEOS) and $\mathrm{O}_{2}$ as precursors. A final $\mathrm{HCl}$ dip $\left(3 \mathrm{~mol} \cdot \mathrm{l}^{-1}\right)$ immediately before $\mathrm{SiO}_{2}$ deposition is used to achieve a clean $\mathrm{Ge}_{0.95} \mathrm{Sn}_{0.05} / \mathrm{SiO}_{2}$ interface. Contact holes are defined using optical lithography and a two-step etching process. First, $\mathrm{CHF}_{3} \mathrm{RIE}$ in a parallel plate reactor is used with $50 \mathrm{~nm}$ of $\mathrm{SiO}_{2}$ remaining in the contact holes after dry etching. The remaining $\mathrm{SiO}_{2}$ is then opened wet-chemically using buffered hydrofluoric acid to avoid overetching and roughening of the $\mathrm{Ge}_{0.95} \mathrm{Sn}_{0.05}$ in the contact holes. For electrical contacts, $\mathrm{Al}$ with thickness of $1.2 \mu \mathrm{m}$ is deposited using magnetron sputtering. The electrical contacts are defined using optical lithography and wet chemical etching. For wet chemical etching, a standard $\mathrm{Al}$ etching solution containing $\mathrm{H}_{3} \mathrm{PO}_{4}, \mathrm{HNO}_{3}$ and $\mathrm{HCOOH}$ is used. The finished device is shown in Fig. 1 (c).

\section{Appendix B: Optical orientation in GeSn}

We exploit a full-zone 30-band $\mathbf{k} \cdot \mathbf{p}$ model to calculate the band structure of GeSn and to obtain the spinpolarization at the generation time. The model is based on Ref. 33 with properly adjusted parameters [41]. We exploit $\approx 380 \times 10^{3} \mathbf{k}$ points to map the reduced Brillouin zone, with a finest region around $\Gamma$. The calculated band structure of GeSn with $5 \%$ of Sn content is reported in Fig. 3 (a). 


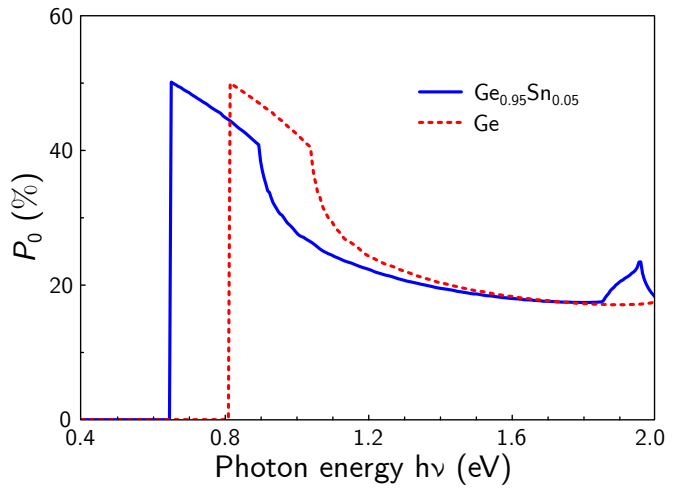

Figure 5. (color online) Calculated optical spin polarization of GeSn (blue continuous line) and Ge (red dotted line).

We follow the model of Ref. 34 to calculate the spinpolarization at the generation time of GeSn. To validate the calculations we compare our results with $0 \%$ of Sn content, i.e., pure Ge, with the results of Ref. 34, the two being in good agreement. The spin-polarizations at the generation time are reported in Fig. 5. The calculated spin-polarization of GeSn results to be the same of $\mathrm{Ge}$, provided that everything is shifted in the energy spectrum to follow the varitions of bandgap produced by alloying.

\section{Appendix C: Calculation of spin current density}

To calculate the spatial average along the stripe thickness of the steady-state spin current densities $j_{\mathrm{s}}^{i}$ in the generic $i^{\text {th }}$ valley, we solve the coupled spin continuity and diffusion equations [42]:

$$
\begin{gathered}
\frac{\partial j_{\mathrm{s}}^{i}(z)}{\partial z}=-\frac{n_{i}(z)}{\tau_{\mathrm{s}}^{i}}+P_{0}^{i} \alpha \Phi_{\mathrm{ph}} e^{-\alpha z} \\
j_{\mathrm{s}}^{i}(z)=-D_{\mathrm{e}} \frac{\partial n_{i}}{\partial z}
\end{gathered}
$$

where $n_{i}, \tau_{i}$ and $P_{0}^{i}$ are the spin-polarized electron population, spin lifetime and spin-polarization at the generation time of the $i^{\text {th }}$ valley, respectively. $\alpha$ is the absorption coefficient and $D_{\mathrm{e}} \approx 1.27 \mathrm{~cm}^{2} / \mathrm{s}$ the diffusion coefficient. We solve Eq. (C1) by imposing the boundary conditions $j_{\mathrm{s}}^{i}\left(z=0, d_{z}\right)=0$, which account for the impossibility for spins to leave the stripe at the GeSn/air $(z=0)$ and $\mathrm{GeSn} / \mathrm{Si}\left(z=d_{z}\right)$ interfaces. The value $j_{\mathrm{s}}^{i}$ results from the spatial average of $j_{\mathrm{s}}^{i}(z)$ over the whole stripe thickness $d_{z}$.

It should be noticed that in Eq. (C1b) the drift term $-\mu n_{i} E_{z}$ does not appear, being $E_{z}$ the electric field along the $z$-axis. Anyway we demonstrate that a finite-element method model accounting for both the presence of the $\mathrm{Si}$ substrate and of the built-in electric field of the junction (see Ref. 38 for details) does not provide for any noticeable difference with respect to the one proposed in the present work.
[1] D. D. Awschalom and M. E. Flattè, Challenges for semiconductor spintronics, Nature Physics 3, 153 (2007).

[2] J. Fabian, A. Matos-Abiague, C. Ertler, P. Stano, and I. Žutić, Semiconductor spintronics, Acta Physica Slovaca. Reviews and Tutorials 57, 10.2478/v10155-0100086-8 (2007).

[3] K. Ando and E. Saitoh, Observation of the inverse spin Hall effect in silicon, Nature Communications 3, 629 (2012).

[4] F. Bottegoni, C. Zucchetti, G. Isella, E. Pinotti, M. Finazzi, and F. Ciccacci, Modeling the photo-induced inverse spin-hall effect in pt/semiconductor junctions, Journal of Applied Physics 124, 033902 (2018).

[5] F. Bottegoni, C. Zucchetti, F. Ciccacci, M. Finazzi, and G. Isella, Optical generation of pure spin currents at the indirect gap of bulk si, Applied Physics Letters 110, 042403 (2017).

[6] F. Bottegoni, M. Celebrano, M. Bollani, P. Biagioni, G. Isella, F. Ciccacci, and M. Finazzi, Spin voltage generation through optical excitation of complementary spin populations, Nature Materials 13, 790 (2014).

[7] C. Zucchetti, F. Bottegoni, G. Isella, M. Finazzi, F. Rortais, C. Vergnaud, J. Widiez, M. Jamet, and F. Ciccacci, Spin-to-charge conversion for hot photoexcited electrons in germanium, Physical Review B 97, 125203 (2018).
[8] F. Bottegoni, C. Zucchetti, G. Isella, M. Bollani, M. Finazzi, and F. Ciccacci, Spin-charge interconversion in heterostructures based on group-IV semiconductors, La Rivista del Nuovo Cimento 10.1007/s40766-020-0002-0 (2020).

[9] T. Guillet, C. Zucchetti, Q. Barbedienne, A. Marty, G. Isella, L. Cagnon, C. Vergnaud, H. Jaffrès, N. Reyren, J.-M. George, A. Fert, and M. Jamet, Observation of large unidirectional rashba magnetoresistance in ge(111), Phys. Rev. Lett. 124, 027201 (2020).

[10] C. Zucchetti, M.-T. Dau, F. Bottegoni, C. Vergnaud, T. Guillet, A. Marty, C. Beigné, S. Gambarelli, A. Picone, A. Calloni, G. Bussetti, A. Brambilla, L. Duò, F. Ciccacci, P. K. Das, J. Fujii, I. Vobornik, M. Finazzi, and M. Jamet, Tuning spin-charge interconversion with quantum confinement in ultrathin bismuth films, Physical Review B 98, 184418 (2018).

[11] T. Guillet, C. Zucchetti, A. Marchionni, A. Hallal, P. Biagioni, C. Vergnaud, A. Marty, H. Okuno, A. Masseboeuf, M. Finazzi, F. Ciccacci, M. Chshiev, F. Bottegoni, and M. Jamet, Spin orbitronics at a topological insulator-semiconductor interface, Phys. Rev. B 101, 184406 (2020).

[12] C. Zucchetti, F. Bottegoni, C. Vergnaud, F. Ciccacci, G. Isella, L. Ghirardini, M. Celebrano, F. Rortais, A. Fer- 
rari, A. Marty, M. Finazzi, and M. Jamet, Imaging spin diffusion in germanium at room temperature, Physical Review B 96, 014403 (2017).

[13] C. Zucchetti, M. Bollani, G. Isella, M. Zani, M. Finazzi, and F. Bottegoni, Doping dependence of the electron spin diffusion length in germanium, APL Materials 7, 101122 (2019).

[14] K. Hamaya, Y. Fujita, M. Yamada, M. Kawano, S. Yamada, and K. Sawano, Spin transport and relaxation in germanium, Journal of Physics D: Applied Physics 51, 393001 (2018).

[15] C. Zucchetti, A. Ballabio, D. Chrastina, S. Cecchi, M. Finazzi, M. Virgilio, G. Isella, and F. Bottegoni, Probing the in-plane electron spin polarization in ge/ $\mathrm{si}_{0.15} \mathrm{ge}_{0.85}$ multiple quantum wells, Phys. Rev. B 101, 115408 (2020).

[16] P. Moontragoon, Z. Ikonić, and P. Harrison, Band structure calculations of si-ge-sn alloys: achieving direct band gap materials, Semiconductor Science and Technology 22, 742 (2007).

[17] S. Wirths, D. Buca, and S. Mantl, Si-ge-sn alloys: From growth to applications, Progress in Crystal Growth and Characterization of Materials 62, 1 (2016).

[18] M. Bauer, C. Ritter, P. A. Crozier, J. Ren, J. Menendez, G. Wolf, and J. Kouvetakis, Synthesis of ternary sigesn semiconductors on $\mathrm{si}(100)$ via $\mathrm{sn}_{x}$ ge $_{1-x}$ buffer layers, Applied Physics Letters 83, 2163 (2003).

[19] S. Wirths, R. Geiger, N. Von Den Driesch, G. Mussler, T. Stoica, S. Mantl, Z. Ikonic, M. Luysberg, S. Chiussi, J. M. Hartmann, H. Sigg, J. Faist, D. Buca, and D. Grützmacher, Lasing in direct-bandgap GeSn alloy grown on Si, Nature Photonics 9, 88 (2015).

[20] H. S. Mạczko, R. Kudrawiec, and M. Gladysiewicz, Material gain engineering in $\mathrm{GeSn} / \mathrm{Ge}$ quantum wells integrated with an Si platform, Scientific Reports 6, 34082 (2016).

[21] F. Pezzoli, A. Giorgioni, D. Patchett, and M. Myronov, Temperature-Dependent Photoluminescence Characteristics of GeSn Epitaxial Layers, ACS Photonics 3, 2004 (2016), arXiv:1609.00542.

[22] F. Schwierz, J. Pezoldt, and R. Granzner, Twodimensional materials and their prospects in transistor electronics, Nanoscale 7, 8261 (2015).

[23] L. Jin, H. Zhu, D. Zhang, B. Liu, H. Meng, X. Tang, M. Li, Z. Zhong, and H. Zhang, Spin pumping and laser modulated inverse spin hall effect in yttrium iron garnet/germanium heterojunctions, Applied Physics Letters 116, 122405 (2020).

[24] S. De Cesari, A. Balocchi, E. Vitiello, P. Jahandar, E. Grilli, T. Amand, X. Marie, M. Myronov, and F. Pezzoli, Spin-coherent dynamics and carrier lifetime in strained $\mathrm{ge}_{1-x} \mathrm{sn}_{x}$ semiconductors on silicon, Phys. Rev. B 99, 035202 (2019).

[25] G. Lampel, Nuclear dynamic polarization by optical electronic saturation and optical pumping in semiconductors, Physical Review Letters 20, 491 (1968).

[26] D. T. Pierce and F. Meier, Photoemission of spinpolarized electrons from GaAs, Physical Review B 13, 5484 (1976).

[27] F. Meier and B. P. Zakharchenya, Optical Orientation (Elsevier, 1984).

[28] M. Dyakonov and V. Perel, Possibility of orienting electrons spins with current, JETP Letters 13, 467 (1971).
[29] M. Dyakonov and V. Perel, Current-induced spin orientation of electrons in semiconductors, Physics Letters A 35, 459 (1971).

[30] SuperK Extreme EXW-12, NKT Photonics. The laser delivers nanosecond pulses at a $78 \mathrm{MHz}$ repetition rate. Here, we do not exploit the temporal structure of the laser source. Indeed, our results are time-averaged since the temporal average of the time-dependent spin driftdiffusion equation equals the solution for the spin driftdiffusion equation in the steady-state, see Ref. 38.

[31] C. Zucchetti, A. Ballabio, D. Chrastina, S. Cecchi, M. Finazzi, M. Virgilio, G. Isella, and F. Bottegoni, Probing the in-plane electron spin polarization in ge/ $\mathrm{si}_{0.15} \mathrm{ge}_{0.85}$ multiple quantum wells, Phys. Rev. B 101, 115408 (2020).

[32] K. Ando, M. Morikawa, T. Trypiniotis, Y. Fujikawa, C. H. W. Barnes, and E. Saitoh, Direct conversion of light-polarization information into electric voltage using photoinduced inverse spin-Hall effect in $\mathrm{Pt} / \mathrm{GaAs}$ hybrid structure: Spin photodetector, Journal of Applied Physics 107, 113902 (2010).

[33] Z. Song, W. Fan, C. S. Tan, Q. Wang, D. Nam, D. H. Zhang, and G. Sun, Band structure of ge1-x sn x alloy: a full-zone 30-band k · p model, New Journal of Physics 21, 073037 (2019).

[34] J. Rioux and J. E. Sipe, Optical injection and control in germanium: Thirty-band $\mathbf{k} \cdot \mathbf{p}$ theory, Physical Review B 81, 155215 (2010).

[35] C. Guite and V. Venkataraman, Temperature dependence of spin lifetime of conduction electrons in bulk germanium, Applied Physics Letters 101, 252404 (2012).

[36] N. Okamoto, H. Kurebayashi, T. Trypiniotis, I. Farrer, D. a. Ritchie, E. Saitoh, J. Sinova, J. Mašek, T. Jungwirth, and C. H. W. Barnes, Electric control of the spin Hall effect by intervalley transitions, Nature Materials 13, 932 (2014).

[37] P. Mudi, S. K. Khamari, and T. K. Sharma, Role of hot electrons in the development of gaas-based spin hall devices with low power consumption, Physica Status Solidi - Rapid Research Letters 14, 2000097 (2020).

[38] C. Zucchetti, G. Isella, F. Ciccacci, M. Finazzi, and F. Bottegoni, Spin transport and spin-charge interconversion phenomena in Ge-based structures, in Spintronics XII, Vol. 11090, edited by H.-J. M. Drouhin, J.-E. Wegrowe, and M. Razeghi, International Society for Optics and Photonics (SPIE, 2019) pp. $73-82$.

[39] E. Kasper, M. Bauer, and M. Oehme, Quantitative secondary ion mass spectrometry analysis of sio2 desorption during in situ heat cleaning, Thin Solid Films 321, 148 (1998).

[40] D. V. Yurasov, A. V. Antonov, M. N. Drozdov, V. B. Schmagin, K. E. Spirin, and A. V. Novikov, Antimony segregation in ge and formation of n-type selectively doped ge films in molecular beam epitaxy, Journal of Applied Physics 118, 145701 (2015).

[41] As suggested by a private communication with Dr. Fan, we exploit the following values of $P_{1}$ and $Q_{2}$ parameters: $\quad P_{1}=1.1701+0.4655 x-0.4649 x^{2}$, $Q_{2}=-0.7412-2.0669 x+3.4247 x^{2}$, being $x$ the fraction of Sn content in the GeSn alloy.

[42] G. Isella, F. Bottegoni, A. Ferrari, M. Finazzi, and F. Ciccacci, Photon energy dependence of photo-induced inverse spin-hall effect in pt/gaas and pt/ge, Applied Physics Letters 106, 232402 (2015). 